\title{
A Note on the Convolution Scandal
}

\author{
Irwin W. Sandberg
}

\begin{abstract}
A recent proposed example of a discrete-time linear time-invariant system that has no convolution representation is discussed in relation to a certain two-term representation for linear systems that are continuous and take bounded inputs into bounded outputs. It is observed that the example lacks a key property, but that the example can be transformed into one that possesses the property.
\end{abstract}

Index Terms-BIBO systems, convolution sums, linear systems.

\section{Two-TERM REPRESENTATION AND THE EXAMPLE}

I $\mathrm{N}$ THIS LETTER, a recent proposed example of a linear time-invariant system that has no convolution representation is discussed in relation to a certain two-term representation for linear systems that are continuous and take bounded inputs into bounded outputs. It is observed that the example lacks a key property, but that the example can be transformed into one that possesses the property. We begin with background material and a description of the two-term representation.

The most important single proposition in the theory of discrete-time single-input single-output linear systems, with realvalued inputs and outputs, is the idea that every such system has an input-output map $H$ that can be represented by an expression of the form

$$
(H x)(n)=\sum_{p=-\infty}^{\infty} h(n, p) x(p)
$$

in which $n$ takes values in the set of integers, $x$ is the input, and $h$ is the system function associated with $H$ in a certain familiar way. It is widely known that this, and a corresponding representation for time-invariant systems in which $h(n, p)$ is replaced with $h(n-p)$, are discussed in many books (see for example [1], pp. 267-269), ([2], pp. 77-79), or ([3], pp. 66-71). Almost always it is emphasized that these representations hold for all linear input-output maps $H$. In [4] ${ }^{1}$ we direct attention to the fact that such statements are in error and we give a correct representation in which an additional term is added to the right side of (1). This writer does not claim that $H$ s for which the additional term is needed are necessarily of importance in applications, but

\footnotetext{
Manuscript received November 3, 2000. The associate editor coordinating the review of this manuscript and approving it for publication was Prof. A. M. Sayeed.

The author is with the Department of Electrical and Computer Engineering, University of Texas, Austin, TX 78712 USA (e-mail: sandberg@ece.utexas.edu).

Publisher Item Identifier S 1070-9908(01)05242-7.

${ }^{1} \mathrm{~A}$ conference version appears in Appendix $\mathrm{G}$ of [5]. [5] contains selected papers given at the 4th Bayona Workshop on Intelligent Methods for Signal Processing and Communications, Bayona, Spain, June 1996.
}

he does feel that their existence shows that the analytical ideas in the books are flawed. ${ }^{2}$

More specifically, it is shown in [4] that

$$
(H x)(n)=\sum_{p=-\infty}^{\infty} h(n, p) x(p)+\lim _{k \rightarrow \infty}\left(H E_{k} x\right)(n)
$$

for each $n$, in which $h$ has the same meaning as in (1), and $E_{k} x$ denotes the function given by $\left(E_{k} x\right)(p)=x(p)$ for $|p|>k$ and $\left(E_{k} x\right)(p)=0$ otherwise. This holds whenever the input set is the set of bounded functions, the outputs are bounded, and $H$ is continuous (with respect to the usual sup norm). In particular, we see that in this setting, an $H$ has a representation of the form given by (1) if and only if

$$
\lim _{k \rightarrow \infty}\left(H E_{k} x\right)(n)=0
$$

for all $x$ and $n .^{3}$ Since this is typically a very reasonable condition for a system map $H$ to satisfy, it is clear that the $H$ s that cannot be represented using just (1) are rather special.

It is an interesting fact that at the present time, the known examples of $H \mathrm{~s}$ for which the additional term is not always zero are not simple, in that they have been shown to exist, but have not been explicitly exhibited. ${ }^{4}$ On the other hand, the map $M$ given by

$$
(M x)(n)=\lim _{m \rightarrow \infty} \frac{1}{2 m+1} \sum_{k=-m}^{m} x(k)
$$

is cited in [16] as an example of a linear time-invariant system that cannot be represented by a convolution, the point being that $M$ s impulse response is the zero function, while the (constant function) output $M x$ need not be the zero function for all inputs $x$. As indicated in [16], a closely related earlier proposed example is given in [12].

There are many other examples of linear time-invariant maps whose impulse response is the zero function, and whose re-

\footnotetext{
${ }^{2}$ The oversight in the books is due to the lack of validity of the interchange of the order of performing a certain infinite sum and then applying $(H \cdot)(n)$. The infinite sum at issue clearly converges pointwise, but that is not enough to justify the interchange. From another viewpoint, the oversight is due to the invalid conclusion (see [6]) that superposition always holds in the case of a countably infinite number of excitations.

${ }^{3}$ The main result in [4] is actually more general in that $H$ s are addressed for which inputs and outputs depend on an arbitrary finite number of variables. As is well known, that case is of interest in connection with, for instance, image processing. A recent paper [15] contains a result corresponding to (2) for the case of linear discrete-space systems with stochastic inputs.

${ }^{4} \mathrm{~A}$ result in ([8, p. 58]) provides such an example. This is observed in [9]. See also [4] or [5], and for related studies, see [10]-[12]. It appears that as early as 1932, Banach was aware of the lack of existence of generalized-convolution representations for certain linear system maps (see [13, pp. 158-159]). For related material in the context of the theory of conjugate spaces, see e.g., [14, p. 228, Table I and p. 229, Exercise 9].
} 
sponse need not be the zero function for all inputs. For example, $M$ given by

$$
(M x)(n)=\lim _{k \rightarrow-\infty} x(k)
$$

will do. However, note that one does not obtain an example of an $H$ for which the additional term in (2) is not always zero by just taking $H$ to be the $M$ of (3) or (4), because in neither case is $M$ even defined on the set of all bounded inputs $x$. Thus, (3) and (4) do not illustrate the important fact that the extra term in (2) can be nonzero, because (2) addresses the much more familiar case of linear systems that take the set of all bounded inputs into bounded outputs, with outputs depending continuously on inputs.

But this does not mean that the observations concerning (3) and (4) are not useful in the context of $H$ s. In fact, with $K$ denoting the map $M$ of either (3) or (4), using a result concerning the extension of maps given in the Appendix, it follows directly that there exists a linear shift-invariant map $H$ that extends $K$ to the set of all bounded inputs, and which provides an example of an $H$ for which the additional term is not always zero. By $H$ extending $K$, we mean that $H x=K x$ for all bounded $x$ for which $K x$ is defined. The result in the Appendix is applicable because of the following.

i) The set of bounded $x$ s for which $K x$ is defined can be seen to be a linear manifold $L_{K}$ in the space $\ell_{\infty}$ of all bounded inputs with the usual sup norm $\|\cdot\| .{ }^{5}$

ii) The linear manifold $L_{K}$ is closed under translation (in the sense described in the Appendix), and $K$ is shift invariant. ${ }^{6}$

iii) The map $K$ is bounded in the sense that $\|K x\| \leq\|x\|$ for $x \in L_{K}$, implying (by a standard result concerning the relation between continuity and boundedness) that $K$ is continuous as a map from $L_{K}$ to $\ell_{\infty}$.

\section{APPENDIX \\ THE EXTENSION PROPOSITION}

Let $\ell_{\infty}$ be the normed linear space mentioned toward the end of Section I. Let $L$ denote a linear manifold in $\ell_{\infty}$ that is closed under translation in the sense that $T_{m} L=L$ for each $m \in \mathcal{Z}$, where $\mathcal{Z}$ is the set of integers and $T_{m}$ is the usual shift map defined on $L$ for each $m$ by $\left(T_{m} x\right)(n)=x(n-m), n \in \mathcal{Z}$. We do not rule out the possibility that $L=\ell_{\infty}$.

Let $A$ be a linear map of $L$ into $\ell_{\infty}$. Such an $A$ is shift-invariant if

$$
(A x)(n-m)=\left(A T_{m} x\right)(n), \quad n \in \mathcal{Z}
$$

${ }^{5}$ Recall that a linear manifold in $\ell_{\infty}$ is just a subset of $\ell_{\infty}$ that is a linear space.

${ }^{6}$ With regard to ii) and $K=M$ of (3), one can check that for each integer $p$ and each bounded $x$ for which $\lim _{m \rightarrow \infty}(2 m+1)^{-1} \sum_{k=-m}^{m} x(k)$ exists, the limit $\lim _{m \rightarrow \infty}(2 m+1)^{-1} \sum_{k=-m}^{m} x(k+p)$ also exists, and the two limits are equal. for each $m \in \mathcal{Z}$ and $x \in L$. It is bounded if $\|A\|_{L}:=$ $\sup \{\|A x\|: x \in L,\|x\| \leq 1\}<\infty$, in which (as before) $\|\cdot\|$ is the norm in $\ell_{\infty}$. Our result is the following.

Proposition: Let $A$ be shift invariant and bounded. Then there exists a bounded (and thus continuous) linear shift-invariant map $B$ from $\ell_{\infty}$ into itself that extends $A$ in the sense that $B x=A x, x \in L$.

The proof of the proposition is similar to a proof in [4], and by the shift invariance of $A$, we have $(A x)(n)=\left(A T_{-n} x\right)(0)$ for all $n$ and all $x \in L$. The map $(A \cdot)(0)$ is a bounded linear functional on $L$, because

$$
\begin{aligned}
& |(A y)(0)| \\
& \quad=\left|\left(A T_{-n} T_{n} y\right)(0)\right|=\left|\left(A T_{n} y\right)(n)\right| \\
& \quad \leq \sup _{\beta}\left|\left(A T_{n} y\right)(\beta)\right| \leq\|A\|_{L} \cdot\left\|T_{n} y\right\|=\|A\|_{L} \cdot\|y\|
\end{aligned}
$$

for $y \in L$. By the Hahn-Banach theorem ([14, p. 178]) there is a bounded linear functional $\mathcal{F}$ that extends $(A \cdot)(0)$ to all of $\ell_{\infty}$. Define $B$ on $\ell_{\infty}$ by $(B x)(n)=\mathcal{F} T_{-n} x$. It is easy to check that $B$ is a linear shift-invariant bounded map into $\ell_{\infty}$, and that $B$ extends $A$ to $\ell_{\infty}$. This completes the proof.

\section{REFERENCES}

[1] W. M. Seibert, Circuits, Signals, and Systems. Cambridge, MA: MIT, 1997.

[2] A. V. Oppenheim, A. S. Willsky, and S. H. Nawab, Signals and Systems. Upper Saddle River, NJ: Prentice-Hall, 1997.

[3] J. G. Proakis and D. G. Manolakis, Digital Signal Processing. New York: Macmillan, 1992.

[4] I. W. Sandberg, "A representation theorem for linear discrete-space systems," Math. Probl. Eng., vol. 4, no. 5, pp. 369-375, 1998.

[5] - "Multidimensional nonlinear myopic maps, Volterra series, and uniform neural-network approximations," in Intelligent Methods in Signal Processing and Communications, D. Docampo, A. Figueiras-Vidal, and F. Perez-Gonzalez, Eds. Boston, MA: Birkhauser, 1997, pp. 99-128.

[6] — , "The superposition scandal," J. Circuits, Syst., Signal Process., vol. 17 , no. 6, pp. 733-735, 1998.

[7] — "Notes on representation theorems for linear discrete-space systems," in Proc. Int. Symp. Circuits and Systems, Orlando, FL, May 30-June 21999.

[8] L. V. Kantorovich and G. P. Akilov, Functional Analysis. Oxford, U.K.: Pergamon, 1982.

[9] S. P. Boyd, "Volterra Series: Engineering Fundamentals," Ph.D. dissertation, Univ. California, Berkeley, 1985.

[10] W. J. Borodziewicz, K. J. Jaszczak, and M. A. Kowalski, "A note on mathematical formulation of discrete-time linear systems," Signal Process., vol. 5, pp. 369-375, 1983.

[11] A. P. Kishore and J. B. Pearson, "Kernel representations and properties of discrete-time input-output systems," Lin. Algeb. Applicat., vol. 205-206, pp. 893-908, 1994.

[12] P. Hughett, "Representation theorems for semilocal and bounded linear shift-invariant operators on sequences," Signal Process., vol. 67, pp. 199-209, 1998.

[13] R. E. Edwards, Functional Analysis. New York: Dover, 1995

[14] G. Bachman and L. Narici, Functional Analysis. New York: Academic, 1966.

[15] I. W. Sandberg, "A note on representation theorems for linear discretespace systems with stochastic inputs," Int. J. Circuit Theory Applicat., to be published.

[16] P. J. Ferreira, "Nonlinear systems and exponential eigenfunctions," IEEE Signal Processing Lett., vol. 6, pp. 287-289, Nov. 1999. 